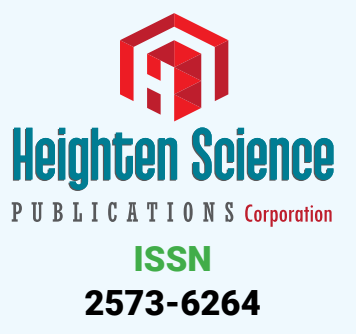

2573-6264

\title{
The short and mid-term effects of Mulligan concept in patients with chronic mechanical neck pain
}

\author{
Konstantinos Zemadanis* \\ PT, MT, MSc student, Experimental Physiology Laboratory, Faculty of Medical School, National \\ and Kapodistrian University of Athens, Greece
}

\begin{abstract}
*Address for Correspondence: Konstantinos Zemadanis, PT, MT, MSc student, Experimental Physiology Laboratory, Faculty of Medical School, National and Kapodistrian University of Athens, Greece, Tel: +302107462592; Email: kostas_zema@yahoo.gr
\end{abstract}

Submitted: 04 April 2018

Approved: 14 April 2018

Published: 16 April 2018

Copyright: @ 2018 Zemadanis K. This is an open access article distributed under the Creative Commons Attribution License, which permits unrestricted use, distribution, and reproduction in any medium, provided the original work is properly cited.

Keywords: Mechanical neck pain; Chronic; Manipulation; Mobilization; Mulligan concept; SNAG; NAG; Self-SNAG

\section{Abstract}

Background: Mechanical chronic neck pain is very common musculoskeletal dysfunction among people, manifesting one or more pain-induced movements and disability impairments. Clinical guidelines suggest passive cervical mobilization and thoracic manipulation as manual therapy interventions. Mulligan concept has positive effect in patients with lumbar and thoracic spine mechanical chronic pain. Study objective was to investigate possible clinical effects of Mulligan techniques in patients with cervical pain according to pain and disability status.

Methods: forty participants diagnosed with mechanical chronic cervical pain, randomly assigned into experimental and control group. NAG, SNAG and self-SNAG joint mobilization in a nine-sessions protocol implemented to the study group, while SHAM-Mulligan techniques applied to control group. Self-reported questionnaires Numeric Pain Rating scale (NPRT) and Neck Disability Index (NDI) were used for the measurement of pain and disability levels respectively. Possible Interactions among Factors (TIME X GROUP) and simple effects in three-time measurements of pre, post and one-month follow-up concerning NDI and NPRS variables, were detected with Mixed-ANOVA test.

Results: Baseline scores of pain and disability resulted in no differences between groups. A significant Group and Time factors interaction founded and simple main effects analysis showed that Mulligan concept-group had significant improvement in post-intervention NPRT and NDI scores $(p<.001)$, compared to baseline scores. Follow-up also differed compared to post-treatment score $(p<.001)$. SHAM-Mulligan control group had no significant differences in dependent variables at any level of TIME factor ( $p>$.001). Significant differences were found between groups according to second and third measurement phase $(p<.001)$.

Conclusion: Our findings suggest that Mulligan concept techniques improve symptoms of pain and disability in chronic mechanical neck pain patients in short and mid-term effect level.

Summary: mobilization techniques of SNAGs, NAGS and self-SNAGs reduces pain and improve function in patients with mechanical neck pain. Taken into consideration the safety and simplicity of application, future studies are encourage to examine the underline mechanism of action.

\section{Introduction}

It is well known that neck pain is one of the most common musculoskeletal disorders among people, especially in those professionals who are spending most of the time in non-active positions like sitting [1,2]. Epidemiology of neck pain lies in a rate of about two thirds of people at some stage, especially in middle age [3,4]. Global Burden of Disease study, ranked neck pain 4th highest in terms of disability as measured by years lived with disability (YLD), and 21st in terms of overall burden [5]. Mechanical nonspecific cervical pain is of cervical vertebrae movement originated and differentiated from other non-structural and non-anatomical causes [6]. Implicating joint structures related are cervical facets, capsule, cartilage and ligaments, having no specific, identifiable etiology [7-9]. Predisposing factors of chronicity includes sporting or occupational activities, poor posture and psychological stress [3,10-12].

How to cite this article: Zemadanis $\mathrm{K}$. The short and mid-term effects of Mulligan concept in patients with chronic mechanical neck pain. J Nov Physiother Rehabil. 2018; 2: 022-021. https://doi.org/10.29328/journal.jnpr.1001018 
Natural history of symptoms may be stable or recurrent, characterized by periods of improvement followed by periods of worsening [13]. Female sex and prior history of neck pain are the strongest and most consistent risk factors for new-onset neck pain in office workers and the general population [1].

According to classification and diagnostic criteria of neck patient's, four subgroups have been recognized: a) neck pain with mobility deficits, b) with movement coordination impairments, c) with headache, d) with radiating pain [14].

Manual therapy in a form of passive manipulation and mobilization is a main conservative treatment approach and has been incorporated in clinical guidelines for chronic neck pain [1] especially in multimodal protocols that includes exercise [15]. Examining the effect of manual therapy in mechanical chronic neck pain, clinical evidence is of moderate to high quality [16]. However, methodologic heterogeneity of parameters regarding small sample sizes, different comparison groups, and lack of long-term measurements, place the need for larger and high-quality randomized controlled trials [17]. Mulligan therapy in the field of manual therapy consists of functional painless techniques that are included in people with spinal pain patients showing immediate symptoms alleviation $[18,19]$. According to this, it is important to investigate the impact of this concept on a subgroup of patients with mechanical neck pain, based on RCT. Taken the current knowledge into consideration, research hypothesis stated that we expected to find a clinical and statistical difference between groups (GROUP-Factor) and within repeated measures (TIME-factor) of pain and disability score, after the implementation of intervention. The objective of the study was to examine the short and mid-term effect of Mulligan concept NAG, SNAG and selfSNAG joint mobilization techniques in patients with chronic mechanical cervical pain according to pain and functional status.

\section{Methods}

The study design was a parallel group, double-blind controlled experimental trial. The sample consisted of 40 patients aging 20-55 years old, with chronic mechanical neck pain who met the study inclusion criteria. Patients recruited from January to May 2017, from a private physiotherapeutic clinic in collaboration with the Experimental Physiology Laboratory of Medical School Faculty at National and Kapodistrian University of Athens. Inclusion criteria were non-smoker participants, having reproducible nonspecific neck pain with a primary location between the supranuchal line and the first thoracic spinous process, lasting more than 3 months, and a Neck Disability Index (NDI) score more than $20 \%$ or more than 2 points in Numeric Pain Rating Scale (NPRS), [20]. Exclusion criteria were a recent significant trauma (including whiplash), headache, dizziness, vertigo, malignancy, radiculopathy, osteoporosis, myelopathy, fracture, metabolic disease, rheumatoid arthritis, upper limb symptoms, long-term corticosteroid and/or painkiller drug use, history of neck surgery, pregnancy and having undergone a spinal manipulative therapy in the previous 2 months [21,22].

After the initial examination by an orthopedic physician, all patients with clinical features of mechanical neck pain, referred for physiotherapy in a private clinic. An experienced specialist in manual therapy examination and treatment, performed a complete physical evaluation of muscle strength, joint mobility and neurodynamic examination of cervical and cervicothoracic region. Possible upper cervical mobilization limitations were detected with safety tests for posterior, anterior and lateral stability of C0-C3 segments, consisting alar and transversus ligament test, extension and rotation vertebral artery tests from supine and sitting position. Given the fact of absence of red flag signs, no imaging was indicated according to relative guidelines [8]. Provocation and alleviation test, as well as Spurling test and upper limb tension tests were performed as special tests for neurological signs and symptoms [23]. 
All participants randomly allocated in the study and control group, each one of 20 patients via random numbers index cards in sealed opaque envelopes. An examiner experienced in NPRS and NDI measurements performed all measures of pain and functionality status respectively at baseline, after the final session ( $\left.9^{\text {th }}\right)$ and four weeks after the completion of manual therapy protocol. Participants were unaware of the research hypothesis, as well as the examiner blinded to the participants' groups' assignment. Prior to any baseline examination measures, all patients read and signed an informed consent form and filled out a complete self-report demographic questionnaire.

\section{Intervention}

The experimental group followed a Mulligan concept manual therapy intervention consisted of Sustained Natural Apophyseal Glides (SNAGs), Natural Apophyseal Glides (NAGs) and self-SNAGs in patient's sitting position. NAGs consists of passive mid to end range oscillatory mobilizations applied anterio-cranially in plane of joint selected. Direction of force is parallel to highly irritable-grossly restricted cervical facet joints. [19]. Application dosage was set to $2-3 \mathrm{Hertz}$ in three sets of three repetitions. SNAGs are an essential mobilization technique applied in cervical spine and consists of the combination of therapist appropriate sustained accessory zygapophyseal glide and the simultaneously patient active symptomatic movement (rotation, flexion, extension, side bending), but in a full range pain free movement. Overpressure in a pain free manner applied at the end range of motion by the patient. Application dosage was set to six repetitions of three sets, accounted for every painful direction of cervical spine Mulligan.

Self-SNAGs: participants in the study group taught the self-SNAG technique described by Mulligan [18]. Application at the appropriate cervical segment with a face towel guiding the mobilization direction, combined with the restricted and/or painful physiological active patient's movement (rotation, flexion, extension, side bending) in a pain free self-manner. Overpressure implementation at the end of available range of movement completed the technique. Application dosage was set to three repetitions of three sets and two trials performed to familiarize participants prior to self-treatment.

The control group of the same participant's number followed a SHAM Mulligan protocol. Application was set on the same parameters as the experimental group protocol in terms of patient's and therapist's starting position, hand placement, face towel implementation, but without the therapist's mobilization force and/ or mobilization direction [24]. Moreover, according to self-SNAG part of the total mobilization procedure, we taught a SHAM self-SNAG on control group participants applying a three-second sustained pressure at the painful and/or restricted cervical segment, without moving the head, but with the same application dosage as the experimental group (Hall et al., 2007). All participants instructed to keep their usual everyday activity, avoiding movements that would possibly exacerbate their main symptoms. We chose to implement only the manual therapy form, without other conservative therapeutic procedure, as it is exercise or any other thermo-hydroelectrotherapy intervention, or even a different manual joint and/or muscle technique, to ensure the individual response of Mulligan concept in our patients' clinical outcome.

An eight year experienced manual therapist, certified in Mulligan concept, applied NAG, SNAGs and trained the patients in self-SNAG mobilization, but also the SHAM Mulligan techniques. It is important to mention that emphasis was given to both techniques to ensure that patients were unaware of whether they received the active intervention or not. All techniques provided individually in patient's restricted and/ or painful movement of flexion, extension, rotation and side bending. For this reason, techniques were preplanned, but the choice of therapeutic direction was pragmatic and individually accordance. Combination of pragmatic-individualized mobilization direction and prescribed-standardized form of manual therapy concept as it is Mulligan 
techniques of SNAGs and NAGs, balances the statistical assumptions of internal/ external validity and generalizability $[25,26]$. Our approach was symptomatic level treated and not randomly chosen. Specific techniques are more effective than general techniques in cervical spine [27].

We chose to implement SNAGs, NAGs and self-SNAGs on sitting weight-bearing position due to functional scope of applications, according to Mulligan concept [19]. We applied nine sessions in a three weeks period $(3 / \mathrm{w})$, with one-day rest between sessions. During the procedure, patients were able to control the movement as actively moved in a pain free range of motion.

\section{Outcome measures}

Measures undertaken at three time phases: baseline, after the intervention and 4 weeks follow-up, by a researcher experienced in collecting information using the selfreport outcome tools of NDI and NPRS, blinded in allocations' group. Another researcher implemented the therapeutic protocol of Mulligan concept and SHAM mobilization.

NDI is the most commonly used patient-reported functional outcome tool [28]. As a validated questionnaire identifies patient's baseline status, monitoring changes relative to pain, function and disability. It consist of ten items, assessing pain/ daily activities and concentration in a score range of " 0 " representing no disability to " 5 " representing complete disability [29]. Structural questionnaire's results are point and percentage (\%) classified: 0-4 (0-8\%) no disability, 5-14 (10-28\%) mild disability, 1524 (30-48\%) moderate disability, 25-34 (50-64\%) severe disability, 35-50 (70-100\%) complete disability. NPRS estimate patient's level of pain. It is a one-dimensional 11-point scale, where patients asked to point out the intensity of current levels of pain over the past 24 hours ranging from 0 (no pain) to 10 (worst pain), [30]. Both instruments showed adequate responsiveness in neck patient population and a fair to moderate test-retest reliability and validity with NDI score of ICC $=0.50,(95 \% \mathrm{CI})$ and NPRS score of ICC $=0.76(95 \% \mathrm{CI})[29,31,32]$. NDI has a minimal detectable change (MDC) of $20 \%$ change and the minimal clinically important difference (MCID) is a $14 \%$ change. The NPRS has an MDC of 2 points and an MCID of 1.3 points in mechanical neck pain patients [32-35].

As clinicians, we use manual contact techniques like joint-play and end-feel, in evaluation of manual therapy effect, but these methods still lacks of evidence based support [36]. External validity is negatively affected from many possible confounding factors in highly controlled research settings investigating motion palpation and manual contact [37]. Lakhani et al., suggest end-feel as a method of monitoring clinical progress [38], but study limitations of small sample size and that only one therapist performed the procedure, awakens the results. On this base, NDI and NPRS are valid and reliable evidence based subjective self-report tools, measuring pain and functional level in neck patents populations [39].

\section{Statistical analysis}

Groups were equal in size, which maximizes the statistical power. The type of variables determined the analysis of the data. Dependent variables were NDI score representing functionality level and NPRS score representing pain level. Independent variables were the two level between-subject factor of GROUP (Mulligan/ SHAM Mulligan) and the three level within subject-factor of TIME (pre/ post/follow up). The effect of Mulligan therapy on pain and functionality examined with 2X3 mixed ANOVA design. Primarily, the main statistical objective was the detection for any interaction between factors of GROUP X TIME, across each of dependent variables. Secondarily, separated ANOVAs performed, based on the simple statistical effects of factors on NPRS and NDI scores, during the three time measures. Confidence interval was set at $95 \%$ and statistical significance at $\mathrm{p}<.05$. In accordance to post hoc analysis, 
planned pairwise comparisons performed, detecting differences among the three levels of within-subjects factor, with Bonferroni correction counteracting for multiple comparisons, reducing the possibility of Type I error. Data analyzed with Statistical Package for Social Sciences 24.0 version (SPSS Inc., Chicago, IL).

Assumptions tests for mixed ANOVA analysis performed, in order to examine the parametric features of the data. Both NDI and NPRS tools are continues variables. Normal distribution of pain and functionality for each factor examined with ShapiroWilk test of normality, which is more appropriate for small sample sizes ( $<50$ samples). Levene's test examined the homogeneity of variances for both the independent variables of pain and functionality. Mauchly's test of sphericity detected the equality variances of within-subject factor due to the three level phases of measurements. Box's $\mathrm{M}$ test detected the equality of dependent variables covariances among groups.

\section{Results}

Sixty-four (64) participants with mechanical neck pain screened for possible eligibility in the study. Of the total number, 40 patients with a mean age of 37 years old and a mean duration of symptoms of 4 months, met the inclusion criteria, randomized into experimental, and control group. Demographics including gender, age, height, weight, drug medication, symptom duration are depicted in table 1 . All patients completed the three week program as well as the three phases of measurements (baseline/after treatment and one-month follow-up). A flow chart according to CONSORT (Consolidated Standards of Reporting Trials; Schulz et al., 2010) indicating flow of participants through each stage of the study, is depicted in figure 1. No adverse effect recorded after Mulligan therapy and SHAM treatment protocol implementation. There were no significant differences in any of the baseline measurement parameters between the experimental and control group. Descriptive statistics of mean and standard deviation (SD) of NPRS and NDI scores of both groups are depicted in tables 2-4].

\begin{tabular}{|c|c|c|}
\hline Table 1: Demographic characteristics of participants in values of Mean, \pm SD. \\
\hline $\begin{array}{c}\text { Group } \\
\text { Sample size, } n\end{array}$ & Mulligan & Control \\
\hline Gender-Female, $n$ & $n=20$ \\
\hline Age years, mean, (SD) & 13 & 15 \\
\hline Symptom duration in months, mean, (SD) & $38( \pm 12.6)$ & $36( \pm 9)$ \\
\hline Weight, kg mean, (SD) & $4.1( \pm 1.1)$ & $4.5( \pm 1.6)$ \\
\hline Height cm, (SD) & $57( \pm 4.7)$ & $61.3( \pm 3.5)$ \\
\hline
\end{tabular}

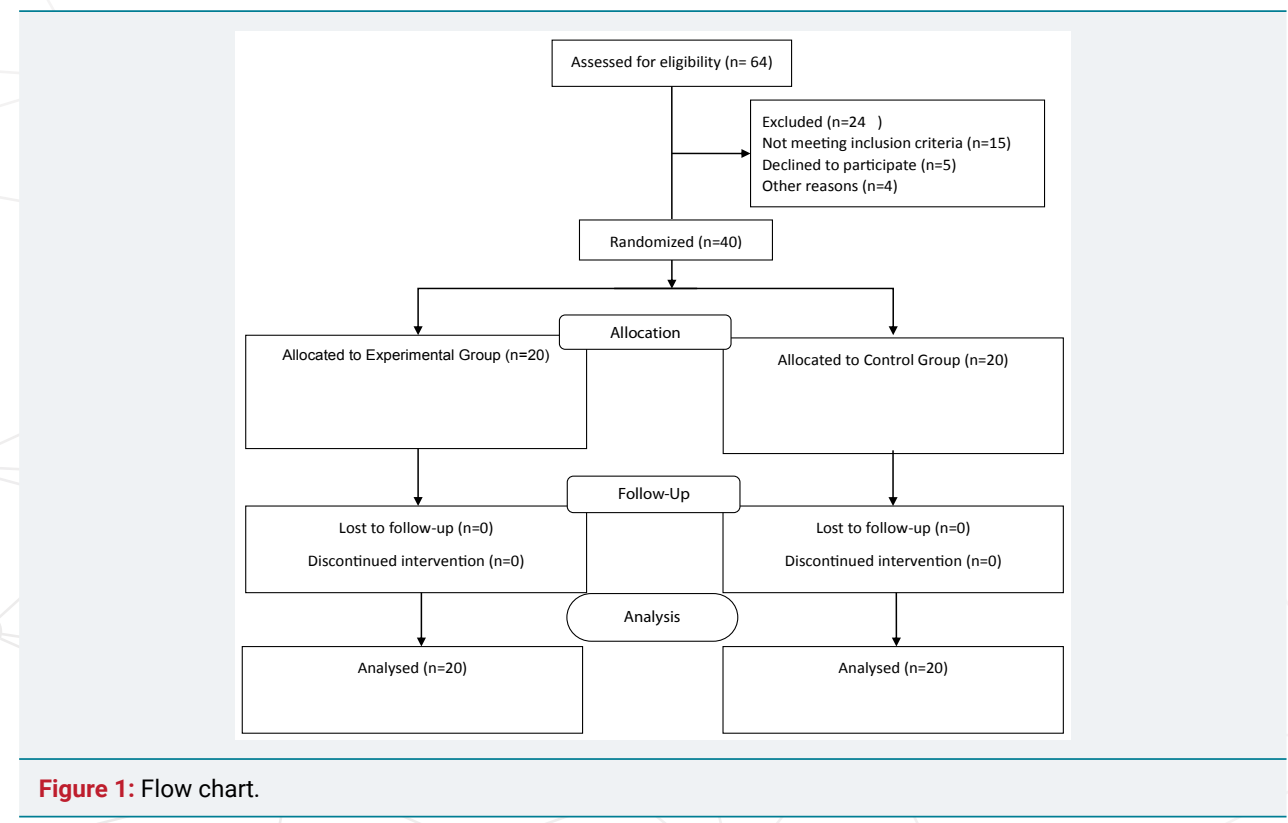


Table 2: NPRT Descriptive statistics (mean, SD) for the two groups in three-time faces: baseline/ post treatment/ follow up.

\begin{tabular}{|c|c|c|c|}
\hline Group & Mean & Std. Deviation & N \\
\hline Baseline Mulligan & 5.0500 & 0.88704 & 20 \\
\hline Baseline Control & 5.3500 & 0.98809 & 20 \\
\hline Post Mulligan & 2.0500 & 0.88704 & 20 \\
\hline Post Control & 4.6000 & 0.99472 & 20 \\
\hline Follow-up Mulligan & 1.5500 & 0.68633 & 20 \\
\hline Follow-up Control & 3.9500 & 0.94451 & 20 \\
\hline
\end{tabular}

Table 3: NDI Descriptive statistics (mean, SD) for the two groups in three-time faces: baseline/ post treatment/ follow up.

\begin{tabular}{|c|c|c|c|}
\hline Group & Mean & Std. Deviation & N \\
\hline Baseline Mulligan & 16.6000 & 5.14424 & 20 \\
\hline Baseline Control & 20.4000 & 3.89872 & 20 \\
\hline Post Mulligan & 8.6000 & 3.56001 & 20 \\
\hline Post Control & 18.8000 & 5.16669 & 20 \\
\hline Follow-up Mulligan & 7.8000 & 3.36546 & 20 \\
\hline Follow-up Control & 18.3500 & 4.63709 & 20 \\
\hline
\end{tabular}

\begin{tabular}{|c|c|c|c|c|c|c|c|}
\hline \multicolumn{2}{|c|}{ GROUP/Mean } & NPRS Pre & NPRS Post & NPRS Follow-up & $\begin{array}{l}\text { NDI } \\
\text { Pre }\end{array}$ & $\begin{array}{l}\text { NDI } \\
\text { Post }\end{array}$ & $\begin{array}{c}\text { NDI } \\
\text { Follow-up }\end{array}$ \\
\hline \multirow{3}{*}{1} & Mulligan & 5.0500 & 2.0500 & 1.5500 & 16.6000 & 8.6000 & 7.8000 \\
\hline & $\mathrm{N}$ & 20 & 20 & 20 & 20 & 20 & 20 \\
\hline & Std. Deviation & 0.88704 & 0.88704 & 0.68633 & 5.14424 & 3.56001 & 3.36546 \\
\hline \multirow{3}{*}{2} & Control & 5.3500 & 4.6000 & 3.9500 & 20.4000 & 18.8000 & 18.3500 \\
\hline & $\mathrm{N}$ & 20 & 20 & 20 & 20 & 20 & 20 \\
\hline & Std. Deviation & 0.98809 & 0.99472 & 0.94451 & 3.89872 & 5.16669 & 4.63709 \\
\hline
\end{tabular}

Mauchly's test of sphericity was non-significant $\mathrm{p}>0.05(\mathrm{p}=0.594)$, assuming that variances of the differences between all possible pairs of the three-level within-subject factor of TIME were equal, according to dependent variable of NPRS. On the contrary, test sphericity founded significant $\mathrm{p}<0.05(\mathrm{p}=0.01)$ according to NDI, therefore a correction was necessary adjusting the degrees of freedom. A Greenhouse-Geisser correction was made with a value of effect size (partial eta square) of $\eta_{\mathrm{p}}{ }^{2}=0.696$. Box's $M$ test was non-significant $p>0.05(p=0.601$ ), assuming the equality of covariances of NPRS score across groups. On the contrary, Box's M test found to be significant $p<0.05$ according to NDI and no covariances equality detected between groups. Levene's test was non-significant, $p>0.05$, assuming the homogeneity of variance of NPRT and NDI scores for both experimental and control group. Shapiro-Wilk test for normality was non-significant ( $p>0.05$ ), resulting in the normal distribution of pain for each group: $\mathrm{p}=0.11$ for Mulligan group and $\mathrm{p}=0.22$ for control group, according to NPRS score. On the side of functionality, the test was also non-significant ( $p>0.05)$, so data was normally distributed for Mulligan ( $p=0.3$ ) and control group $(p=0.28)$ in addition to NDI score.

\section{Interaction ( $F$ value) analysis results}

Mixed ANOVA revealed a significant interaction between factors (GROUP X TIME) of the dependent variables of NPRS with a value of $F(2,76)=42.39$, and NDI with a value of $F(2,76)=35.59,.(p<.001)$. Profile plots of factors interaction are depicted in figures 2 and 3 in non-parallel lines, according to dependent variables of NPRS and NDI respectively.

\section{Simple main effects}

Because of significant factor's interaction, there is a need to follow the simple main effects of each independent variable, meaning that there is a difference (non-paralleled) on their effect on dependent variables. Simple main effect analysis of Group on NPRT and NDI individually was significant $(p<0.05)$, with $F$ values of pain $F(1,38)=53.8$, $(p=0.000)$ and $F$ values for functionality $F(1,38)=41.15,(p=0.000)$, (Tables 5,6$)$. 

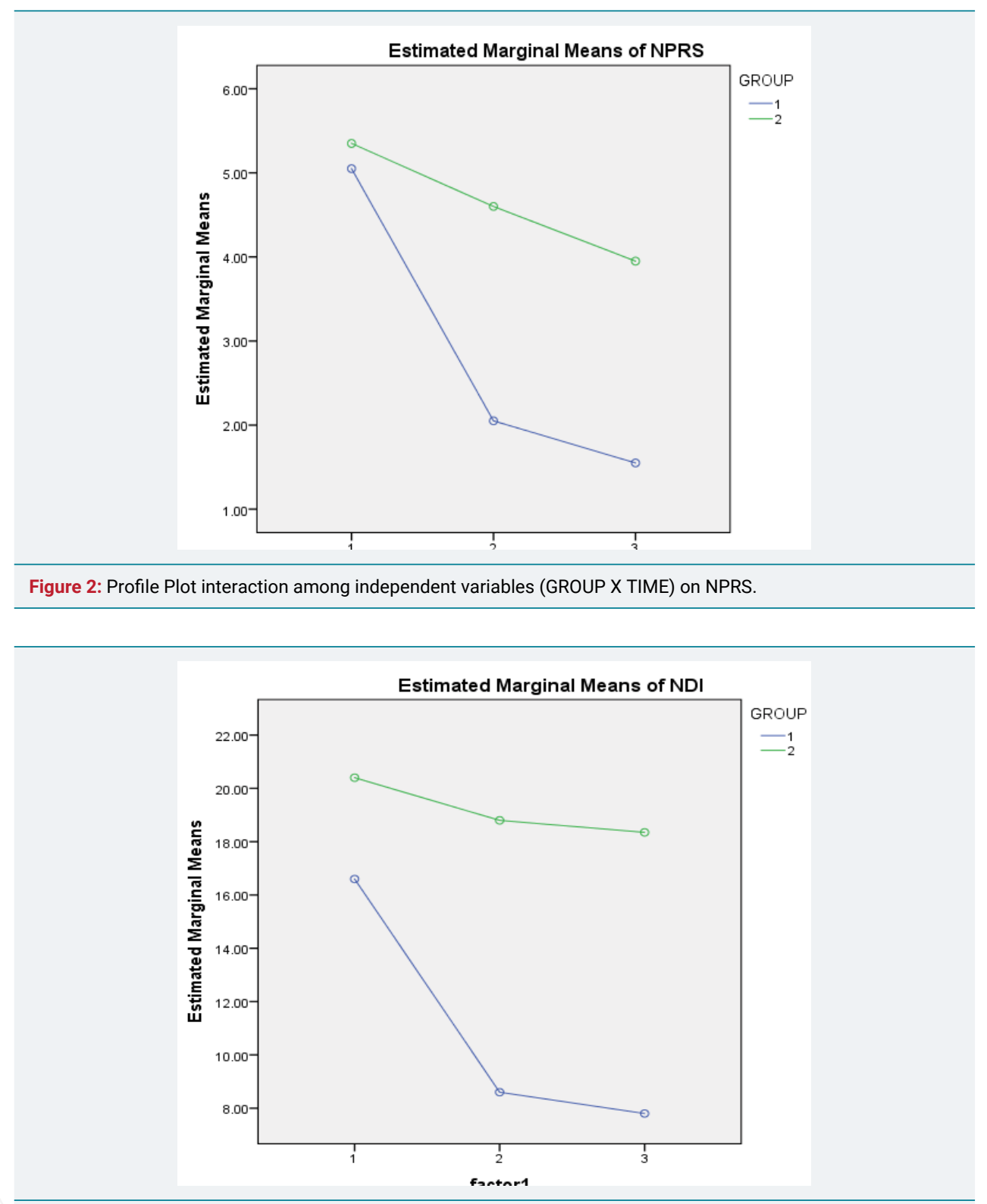

Figure 3: Profile Plot interactions among independent variables (GROUP X TIME) on NDI.

Table 5: Test of Between-Subjects Effects of NPRS

\begin{tabular}{|c|c|c|c|c|c|c|}
\hline Source & Type III Sum of Squares & df & Mean Square & F & Sig. & Partial Eta Squared \\
\hline Intercept & 1695.008 & 1 & 1695.008 & 994.242 & 0.000 & 0.963 \\
\hline Group & 91.875 & 1 & 91.875 & 53.891 & 0.000 & 0.586 \\
\hline Error & 64.783 & 38 & 1.705 & & & \\
\hline
\end{tabular}

Table 6: Test of Between-Subjects Effect of NDI

\begin{tabular}{|c|c|c|c|c|c|c|}
\hline Source & Type III Sum of Squares & df & Mean Square & F & Sig. & Partial Eta Squared \\
\hline Intercept & 27331.008 & 1 & 27331.008 & 559.886 & .000 & .936 \\
\hline Group & 2009.008 & 1 & 2009.008 & $\mathbf{4 1 . 1 5 5}$ & $\mathbf{. 0 0 0}$ & .520 \\
\hline Error & 1854.983 & 38 & 48.815 & & & \\
\hline
\end{tabular}

Simple main effect analysis of TIME factor separately on NPRS and NDI was significant $(\mathrm{p}<0.05)$, with $\mathrm{F}$ values of pain $\mathrm{F}(2,76)=42.39,(\mathrm{p}=0.000)$ and functionality $F(2,76)=35.59, \quad(p=0.000)$. Simple comparisons following significant simple main effects are the next step in our analysis on the three-level TIME factor via tests of within subject's contrasts. Mean differences are significant $(\mathrm{p}<.05)$ among levels of TIME factor for the experimental group as depicted in pairwise comparisons test (Table 7), based on estimated marginal means. 


\begin{tabular}{|c|c|c|c|c|c|}
\hline \multicolumn{7}{|l|}{ Table 7: Experimental group pairwise Comparisons of NPRS. } \\
\hline Mulligan Group & $\mathbf{9 5 \%}$ CI Lower Bound & & \multicolumn{2}{|c|}{$95 \%$ Cl Upper Bound } \\
\hline NPRS post & $1.875^{\star}$ & .125 & .000 & 1.562 & 2.188 \\
\hline NPRS follow up & $2.450^{\star}$ & .140 & .000 & 2.099 & 2.801 \\
\hline NPRS pre & $-1.875^{\star}$ & .125 & .000 & -2.188 & -1.562 \\
\hline NPRS follow-up & $0.575^{\star}$ & .144 & .001 & .214 & .936 \\
\hline NPRS pre & $-2.450^{*}$ & .140 & $\mathbf{0 . 0 0 0}$ & -2.801 & -2.099 \\
\hline NPRS post & $-0.575^{\star}$ & .144 & $\mathbf{0 . 0 0 1}$ & -.936 & -.214 \\
\hline
\end{tabular}

\section{Discussion}

The current study examined the clinical effect of a nine session's series of Mulligan therapy in terms of pain and functionality regarding people with chronic mechanical neck pain. Results showed a significant short and mid-term statistical decrease of pain and function improvement of cervical spine, confirming the initial research hypothesis. The experimental group resulted in significant clinical improvement of post measures comparing baseline, but also in a follow-up measure comparing to post treatment. This was not the case for control group followed a SHAM Mulligan intervention as no any significant difference was found in post and follow up treatment comparing baseline, but also between groups differences. In a similar study, [40] explored the impact of Mulligan concept in neck pain patients, but on a different research design, comparing three groups of SNAG with electrotherapy/self-SNAG with electrotherapy/ electrotherapy alone, resulting in study groups improvements, not only in pain and functionality, but also in joint position sense.

Studies have shown that manual therapy is more effective in multimodal therapeutic protocols [9,41-43]. Effectiveness of manipulation and/or mobilization in isolation for acute or chronic non-specific neck pain remains inconclusive $[42,44,45]$. Our objective was to identify the independent and unilateral Mulligan clinical effect as a monotherapy, without any simultaneously conservative method.

Mulligan techniques have distinguished as a functional approach producing an immediate pain alleviation and Range of Motion (ROM) improvements in chronic musculoskeletal conditions mainly in peripheral joints via MWM [46], but also in lumbar and thoracic spine [19,47]. In cases of successful implementation, patients are encourage to continue the therapeutic protocol adding exercise, reflecting the everyday clinical approach. Therefore, the timing of application is important and evidence demonstrate that early Mulligan mobilization implementation paves the way following exercise application and/or other forms of mobilization [48].

It has been shown that therapeutic effect of manual therapy is in accordance with dosage and duration of therapy [49]. Therefore, in contrast of other studies [50], we conducted a series of sessions avoiding of single intervention protocol, to increase the possible therapeutic impact and reinforce generalizability of clinical findings. A number of nine session or more have been investigated in other studies with positive results [40,51]. Our study design based on the concept of Mulligan therapy effect incorporating all three main components of SNAG/self-SNAG and NAG identified the total clinical impact, against a control group. As already mentioned, we did not include any other conservative intervention, as it is exercise (active, isometric, supervised) and/ or thermo-hydro-electrotherapy therapy.

So far, no adverse effects have been reported in relative studies, concerning Mulligan implementation in the cervical spine and the same was for the current one. This is essential regarding evidence of manipulation's serious effects like stroke, or even death incidence, especially after rotatory High Volume Low Amplitude (HVLA) implementation [52]. Puentendura et al., reported that $44.8 \% \%$ of adverse effects associated with cervical manipulation were characterized as preventable [53], 
excluding all contraindications and red flags. On the contrary, SNAGs technique is a safe application, where patient is able to control the procedure [54]. SNAGs are most successful when symptoms provoked by a movement and are not multilevel, where NAGs indicated in more irritating conditions $[18,55]$.

Self-SNAGs are consider to be a highly indicative therapeutic approach for headache patients, but also in movement restrictions and/ or mechanical pain [54]. Hall, found significant short and long-term improvements in rotation ROM and pain following upper cervical self-SNAG mobilization in headache patients [56]. Similar results recorded in a study examining self-SNAG with exercise over SNAG with the same exercise protocol, concluding that are equally effective in chronic neck pain [39]. However, study design did not compare SNAG alone over a control group as a monotherapy, but exercise implementation was a part of all treatment protocol group.

On the analysis of relevant research of Mulligan concept on mechanical neck pain, no study has ever been examine the clinical effect with no other therapeutic factor on experimental and/or control group. We decided to implicate SHAM SNAG as an alternative control therapy, reducing the effect of confounding factors arising from conservative modalities and placebo effect applications. SHAM mobilization was a part of research protocols accounted for the need of ethical prepositions in clinical trials [22,56,57].

Research results regarding Mulligan therapy and mechanical neck patients are in progress in recent years. Ali et al., found better short-term improvement when SNAG combined with exercise in NDI score, but there was no control group to examine the isolated effect of mobilization [58]. Lopez et al., found similar improvements of manual therapy implementations comparing SNAG [50], HVLA and mobilization, but without using a control group and applying only a single session. In our study, control group incorporated in study design to limit confounding factors arising from natural history of pain. Put et al. showed that Mulligan was superior in terms of ROM [51], comparing with a multimodal therapy consisting of cervical spine massage, electrotherapy and ultrasound in headache patients. Hussain et al, found superiority of NAGS opposite Maitland mobilization in neck patient's measurements of NPRS and NDI, after a fourweek period therapy with a frequency of four session per week [59]. However, both groups were followed an additional parallel protocol of electrotherapy without control group included. In addition, SNAG resulted in better clinical improvements than Maitland in a study by Gautam [60] on the same research hypothesis, including control group, but with a different conventional therapy consisted of exercise and thermotherapy applied to all three groups (SANG/Maitland/control). Ahmad et al., 2013 found better short-term results concerning NDI measurements after Mulligan mobilization [61], in comparison with Kaltenborn mobilization, in non-specific neck patients. Limitations of their study settings were the lack of control group, the absence of detailed report of Mulligan techniques that implemented in participants and also the conventional diathermy and exercise program followed by both experimental group, which could blind the individual effect of mobilization. Another study compared Maitland with exercise, SNAG with exercise and exercise alone in a two weeks protocol with five sessions per week, resulting in no difference in short-term pain, disability and ROM of patients [62]. On the other hand, Abdelgalil et al., found superiority of HVLA in ROM [63], but less pain improvements in comparison with SNAG mobilization. El Sodany et al., 2014 incorporated a one-month follow up ROM [64], VAS and NDI measurements after application of three treatment protocols consisted of SNAG with exercise, manipulation with exercise and exercise alone, on two experimental groups and on the control group respectively. Combined therapy groups resulted in better outcome post and follow-up treatment, but no difference was found between study groups.

The majority of the aforementioned studies implemented a nonstandard application according to the level of cervical spine, guiding by patient's restriction of intervertebral 
movement, based on physical examination. This is in contrast with standard preplanned applications, which can be reproducible, but lack of individual clinical approach, reducing internal validity and increased external validity.

Biomechanical interpretation of Mulligan concept effect still lacks evidence-based support and remaining an intriguing subject. Positional fault theory is unconfirmed [65]. Hearn et al., 2002 proposed mechanisms like facet surfaces separation, releasing the entrapped menisci and stretching adhesions [66]. Neurophysiologic mechanism of action is a more recent hypothesis on a basis of inhibition of nociceptors via stimulation of skin mechanoreceptors resulting from intervertebral joint mobilization [67]. Evidence shows that sympathetic nervous system responses after Mulligan implementation might implicated with neurophysiological variables [68]. On the basis of the above, SNAGs resulted in significant clinical improvements on cervical joint position sense and proprioception in chronic neck pain patients, under the same dosage of our study in week's duration and session's frequency [69]. Moreover, SNAGs, combined with isometric exercise and hot pack application in experimental group, demonstrated significant clinical benefits in parameters of pain, functionality and proprioception, in contrast to control group followed only thermotherapy and exercise. On a different study sample of patients with cervical radiculopathy, SNAG proved an effective therapeutic approach associated with alterations in dermatomal somatosensory evoked potential [70].

Study limitations identified in parameters of small sample size, which reduce the statistical power and the application of more analytical parametrical statistical tests. We did not measure muscle strength and ROM, which could support the clinical effect on NDI and NPRS measures. Patient's sampling conducted at the town of Rio, a relative small area near city of Patras, reducing the generalizability of findings. According to results of our study, recommendations can be made on a more methodologically extended future study settings, in terms of larger sample size, under collaboration with other private clinics where Mulligan concept takes its place in neck patient's therapy. Moreover, different patient's subgroups as it is radiculopathy with upper arm symptoms and/or whiplash injury needs to be incorporated as target study group.

\section{Conclusion}

The current study confirmed the research hypothesis of significant clinical improvements of pain and functionality scores after application of Mulligan concept therapy protocol in patients with chronic mechanical neck pain in short and mid-term level of time measurements. Future studies need to reinforce the current results in more extensive sample of participants as well as in other sub-populations of neck pain patients. Mechanism of action remains unclear, regarding Mulligan clinical interpretation effects. We suggest a more comprehensive and in-depth research regarding biologic parameters on a cellular and molecular level that might be involved.

\section{Acknowledgements}

We are grateful to the staff at the outpatient clinic for their valuable assistant.

\section{References}

1. Blanpied P, Gross A, Elliott J, Devaney L. Neck Pain: Revision 2017. J Orthop Sports Phys Ther. 2017; 47: 1-8.

2. Genebra C, Maciel N, Bento T. Prevalence and factors associated with neck pain: a population-based study. Braz J Phys Ther. 2017; 21: 274-280. Ref.: https://goo.gl/Q48z59

3. Binder A. Neck Pain. Clinical Evidence. 2008; 8: 108-113. Ref.: https://goo.gl/TrFRzs

4. Carroll L, Cassidy J, Peloso P. Methods for the best evidence synthesis on neck pain and its associated disorders: the Bone and Joint Decade 2000-2010 Task Force on Neck Pain and Its Associated Disorders. Spine. 2008; 33: 33-38. Ref.: https://goo.gl/hSb2cf 
5. Hoy D, March L, Woolf A, Blyth F, Brooks $P$, et al. The global burden of neck pain: estimates from the global burden of disease 2010 study. Ann Rheum Dis. 2014; 73: 1309-1315. Ref.: https://goo.gl/vrpppo

6. Cross KM, Kuenze C, Grindstaff TL, Hertel J. Thoracic spine thrust manipulation improves pain, range of motion, and self-reported function in patients with mechanical neck pain: a systematic review. J Orthop Sports Phys. 2011; 41: 633-642. Ref.: https://goo.gl/ErWkcG

7. Bogduk N. The anatomy and pathophysiology of neck pain. Phys Med Rehabil Clin N Am. 2011; 22: 367-382. Ref.: https://goo.gl/jJBxyD

8. Haldeman S, Carroll L, Cassidy JD. Findings from the Bone and Joint Decade 2000 to 2010 Task Force on Neck Pain and Its Associated Disorders. J Occup Environ Med. 2010; 52: 424-427. Ref.: https://goo.gl/nKfxHV

9. Hurwitz E, Carragee E, van der Velde G, Carroll L. Treatment of neck pain: noninvasive interventions: results of the Bone and Joint Decade 2000-2010 Task Force on Neck Pain and Its Associated Disorders. Spine. 2009; 33: 123-152. Ref.: https://goo.gl/a1cx64

10. Clair D, Edmondston S, Allison G. Physical therapy treatment dose for non-traumatic neck pain: a comparison between two patient groups. J Orthop Sports Phys Ther. 2006; 36: 867-875. Ref.: https://goo.gl/Qak6Hv

11. Sarig-Baha H. Evidence for exercise therapy in mechanical neck disorders. Man Ther. 2003; 8: 10-20. Ref.: https://goo.gl/mbF5Wy

12. Paksaichol A, Janwantanakul $P$, Purepong N, Pensri $P$, van der Beek AJ. Office workers' risk factors for the development of non-specific neck pain: a systematic review of prospective cohort studies. Occup Environ Med. 2012; 69: 610-618. Ref.: https://goo.gl/LKfZgM

13. Guzman J, Hurwitz EL, Carroll LJ, et al. A new conceptual model of neck pain: linking onset, course, and care: the Bone and Joint Decade 2000-2010 Task Force on Neck Pain and Its Associated Disorders. J Manipulative Physiol Ther. Spine 2008; 33: S14-23. Ref.: https://goo.gl/3QVQoz

14. Fritz JM, Brennan GP. Preliminary examination of a proposed treatment-based classification system for patients receiving physical therapy interventions for neck pain. Phys Ther. 2007; 87: 513-524. Ref.: https://goo.gl/3QVQoz

15. Kay T, Gross A, Goldsmith C. Exercises for mechanical neck disorders. 2012. Cochrane Database Syst Rev. 2005; 20: CD004250. Ref.: https://goo.gl/uq6H5H

16. Vernon $\mathrm{H}$, Humphreys $\mathrm{K}$, Hagino $\mathrm{C}$. Chronic mechanical neck pain in adults treated by manual therapy: a systematic review of change scores in randomized clinical trials. J Manipulative and Physiological Therapeutics. 2007; 30: 215-227. Ref.: https://goo.gl/2rwRDH

17. Franke H, Franke J, Fryer G. Osteopathic manipulative treatment for chronic nonspecific neck pain A systematic review and meta-analysis. International Journal of Osteopathic Medicine. 2015; 18: 255-267. Ref.: https://goo.gl/e15acB

18. Mulligan BR. Manual Therapy "NAGS", "SNAGS”, “MWMS" etc., 4th edition. Plane View Services Ltd., New Zealand: Wellington. 1999. 1-142.

19. Exelby $L$. The locked lumbar facet joint: intervention using mobilizations with movement. Man Ther. 2001; 6: 116-121. Ref.: https://goo.gl/qrmmND

20. Leaver A, Refshauge K, Maher C, Latimer J. Efficacy of manipulation for non-specific neck pain of recent onset: design of a randomised controlled trial. BMC Musculoskelet Disord. 2007; 8: 18. Ref.: https://goo.gl/qrmmND

21. Griswold D, Learman K, O'Halloran B, Cleland J. A preliminary study comparing the use of cervical/ upper thoracic mobilization and manipulation for individuals with mechanical neck pain. J Man Manip Ther. 2015; 23: 75-83. Ref.: https://goo.gl/2vJSFk

22. Cleland J, Whitman J, Fritz J, Palmer J. Manual physical therapy, cervical traction, and strengthening exercises in patients with cervical radiculopathy: A case series. J Orthop Sports Phys Ther. 2005; 35: 802-811. Ref.: https://goo.gl/yh2wqx

23. Kaltenborn F, Evjenth O, Kaltenborn T, Morgan D, Vollowitz E. Manual mobilization of the joints, vol. II: the spine. 2003. Ref.: https://goo.gl/mdzHaq

24. Moulson A, Watson T. A preliminary investigation into the relationship between cervical snags and sympathetic nervous system activity in the upper limbs of an asymptomatic population. Man Ther. 2006; 11: 214-224. Ref.: https://goo.gl/65Pjuz

25. Masaracchio M, Cleland J, Hellman M, Hagins M. Short-term combined effects of thoracic spine thrust 
manipulation and cervical spine nonthrust manipulation in individuals with mechanical neck pain: a randomized clinical trial. J Orthop Sports Phys Ther. 2013; 43: 118-27. Ref.: https://goo.gl/z8Cv9G

26. Leaver A, Maher C, Herbert R, Latimer J, Mc Auley JH, et al. A randomized controlled trial comparing manipulation with mobilization for recent onset neck pain. Arch Phys Med Rehabil. 2010; 91: 13131318. Ref.: https://goo.gl/v3KEXJ

27. Slaven E, Goode A, Coronado R. The relative effectiveness of segment specific level and non-specific level spinal joint mobilization on pain and range of motion: results of a systematic review and metaanalysis. J Man Manip Ther. 2013; 21: 7-17. Ref.: https://goo.gl/1ywxiH

28. Vernon H. The Neck Disability Index: state-of-the-art, 1991-2008. J Manipulative Physiol Ther. 2008 31: 491-502. Ref.: https://goo.gl/EcGoML

29. Stratford $P$, Riddle D, Binkley J. Using the neck disability index to make decisions concerning individual patients. Physiotherapy Canada. 1999; 51: 107-112.

30. Jensen $M$, McFarland $C$. Increasing the reliability and validity of pain intensity measurement in chronic pain patients. Pain. 1993; 55: 195-203. Ref.: https://goo.gl/Eg2dU9

31. Childs J, Flynn T, Fritz J, Piva S, Whitman J, et al. Screening for vertebrobasilar insufficiency in patients with neck pain: manual therapy decision-making in the presence of uncertainty. $J$ Orthop Sports Phys Ther 2005; 35: 300-306. https://goo.gl/BBsg5j

32. Cleland J, Childs J, Whitman J. Psychometric properties of the Neck Disability Index and Numeric Pain Rating Scale in patients with mechanical neck pain. Arch Phys Med Rehabil. 2008; 89: 69-74. Ref.: https://goo.gl/bojyi9

33. Young S, Aprill C, Braswell J, Ogard W. Psychological factors and domains of neck pain disability. Pain Med. 2009; 10: 310-318. Ref.: https://goo.gl/gZt5W4

34. Pool J, Ostelo R, Hoving J, Bouter L. Minimal clinically important change of the Neck Disability Index and the Numerical Rating Scale for patients with neck pain. Spine. 2007; 32: 3047-3051. Ref.: https://goo.gl/ubie8b

35. Farrar J, Young J, La Moreaux L. Clinical importance of changes in chronic pain intensity measured on an 11-point numerical pain rating scale. Pain. 2001; 94: 149-158. Ref.: https://goo.gl/td1r62

36. Nyberg R, Smith R. The science of spinal motion palpation: a review and update with implications for assessment and intervention. J Man Manip Ther. 2013; 21: 160-167. Ref.: https://goo.gl/RgcbFC

37. Huijbregts P. Spinal motion palpation: a review of reliability studies. J Man Manip Ther. 2002; 10 24-29. Ref.: https://goo.gl/wdb585

38. Lakhani E, Nook B, Haas M, Docrat A. Motion palpation used as a post manipulation assessment tool for monitoring end-feel improvement: a randomized controlled trial of test responsiveness. J Manipulative Physiol Ther. 2009; 32: 549-555. Ref.: https://goo.gl/W1yTFC

39. Desai N, Khatri S, Keerthi R. Are self-SNAGS effective for chronic neck pain? REVISTA ROMÂNĂ DE KINETOTERAPIE. 2012; 18: 22-29.

40. Said S, Ali O, Elazm. Mulligan self-mobilization versus Mulligan SNAG on cervical position sense. Int J Physiother. 2017; 4: 93-100. Ref.: https://goo.gl/fXfPgK

41. Tsakitzidis G, Dankaerts W, Van Royen P. Non-specific neck pain and evidence-based practice. Eur Scientific J. 2013; 9: 1-19.

42. Gross AR, Hoving JL, Haines TA, Goldsmith $\mathrm{CH}$, Kay $\mathrm{T}$, et al. A Cochrane review of manipulation and mobilization for mechanical neck disorders. Spine. 2004; 29: 1541-1548. Ref.: https://goo.gl/DL7BDg

43. Sarigiovannis $P$, Hollins B. Effectiveness of manual therapy in the treatment of non-specific neck pain: a review. Physical Therapy Reviews 2005; 10: 35-50. Ref.: https://goo.gl/Fo7qJJ

44. Gemmell H, Miller P. Comparative effectiveness of manipulation, mobilisation and the Activator instrument in treatment of non-specific neck pain: a systematic review. Chiropr Osteopat. 2006; 14 7. Ref.: https://goo.gl/SGcvrL

45. Cleland J, Flynn T, Childs J. The Audible Pop from Thoracic Spine Thrust Manipulation and Its Relation to Short-Term Outcomes in Patients with Neck Pain. J Manual Manipulative Therapy. 2007; 15: 143-154. Ref.: https://goo.gl/WpLdMa

46. Hing W, Bigelow R, Bremner T. Mulligan's Mobilization with Movement: A Systematic Review J Manual Manipulative Therapy. 2009; 17: 39-66. Ref.: https://goo.gl/NhGK3i 
47. Abdelhay $M$, Elgendy $M$, Lasheen $Y$. Mulligan versus Maitland mobilization on thoracic kyphotic angle in postural kyphosis. Int J Physiother. 2017; 4: 168-172. Ref.: https://goo.gl/1qua28

48. Zemadanis K, Sykaras E, Athanasopoulos S, Mandalidis D. Mobilization-with-movement prior to exercise provides early pain and functionality improvements in patients with patellofemoral pain syndrome. J Int Musculoskeletal Med. 2015; 37: 101-107. Ref.: https://goo.gl/ykXQHi

49. Snodgrass S, Heneghan N, Tsao H, Stanwell P. Recognising neuroplasticity in musculoskeletal rehabilitation: a basis for greater collaboration between musculoskeletal and neurological physiotherapists. Man Ther. 2014; 19: 614-617. Ref.: https://goo.gl/cJgqHk

50. Lopez L, Perez J, Gutierez J. Mobilization versus manipulations versus sustain apophyseal natura glide techniques and interaction with psychological factors for patients with chronic neck pain randomized controlled trial. Eur J Phys Rehabil Med. 2015; 51: 121-32. Ref.: https://goo.gl/KoPSqK

51. Put M, Huber J, Pieniążek M. Randomized Clinical Trial of Multimodal Therapy and Mulligan's Concept of Manual Therapy for Patients with Chronic Pain Syndrome Caused by Upper Cervical Spine Disorders. Int J Orthop Rehabil. 2016; 3: 40-46.

52. Leon-Sanchez A, Cuetter A, Ferrer G. Cervical spine manipulation: an alternative medical procedure with potentially fatal complications. South Med J. 2007; 100: 201-213. Ref.: https://goo.gl/YXwGDZ

53. Puentedura E, Cleland J, Landers M, Mintken P. Development of a clinical prediction rule to identify patients with neck pain likely to benefit from thrust joint manipulation to the cervical spine. J Orthop Sports Phys Ther. 2012; 42: 577-592. Ref.: https://goo.gl/rcXakV

54. Mulligan BR. Manual Therapy NAGS SNAGS MWMS etc. Wellington, New Zealand: Plane View Services; 2004

55. Wilson E. The Mulligan Concept: NAGS, SNAGS, and mobilisations with movement. J Bodywork Movement Therapies. 2001; 5: 81-8.

56. Hall T, Chan H, Christensen L. Efficacy of a C1-C2 Self-sustained Natural Apophyseal Glide (SNAG) in the Management of Cervicogenic Headache. J Orthop Sports Phys Ther. 2007; 37: 100-107. Ref.: https://goo.gl/dM81cU

57. Eui-Ju Shin, Byoung-Hee Lee. The effect of sustained natural apophyseal glides on headache duration and cervical function in women with cervicogenic headache. $J$ Exercise Rehabilitation. 2014; 10: 131-135. Ref.: https://goo.gl/JFyJjc

58. Ali A, Shakil-ur-Rehman S, Sibtain F. The efficacy of Sustained Natural Apophyseal Glides with and without Isometric Exercise Training in Non-specific Neck Pain. Pak J Med Sci. 2014; 30: 872-874. Ref.: https://goo.gl/dLNJ68

59. Hussain H, Ahmad A, Amjad F. Effectiveness of Natural Apophyseal Glides Versus Grade I and II Maitland Mobilization in Non-Specific Neck Pain. Annals. 2016; 22: 1-7. Ref.: https://goo.gl/8Y8sNp

60. Gautam R, Dhamija J, Puri A. Comparison of Maitland and Mulligan mobilization in improving neck pain, ROM and disability. Int J Physiother Res. 2014; 2: 482-487. Ref.: https://goo.gl/oWDGd8

61. Ahmad R, Asim H, Nasir R. Comparison of Mulligan manual therapy techniques with Kaltenborn manual therapy techniques in patients with nonspecific neck pain in improvement on neck disability index. Int J Sci Res. 2015; 4: 1-4.

62. Ganesh S, Mohanty P, Pattnaik M. Effectiveness of mobilization therapy and exercises in mechanical neck pain. Physiother Theory Pract. 2015; 31: 99-106. Ref.: https://goo.gl/mbyYbn

63. Abdelgalil Allam Abdelgalil, Alaa Abdelhakeim Balbaa, Hatem Mohamed Elazizi, Ashraf Abdelaa Mohamed Abdelaal. High Velocity Low Amplitude Manipulation versus Sustained Apophyseal Glides on Pain and Range of Motion in Patients with Mechanical Neck Pain: An Immediate Effect. Int J Adv Res. 2015; 3: 503-513. Ref.: https://goo.gl/U4dRQN

64. El-Sodany AM, Alayat MSM, Zafer AMI. Sustained natural apophyseal glides mobilization versus manipulation in the treatment of cervical spine disorders: a randomized controlled trial. Int $\mathrm{J}$ Adv. 2014; 2: 274-280. Ref.: https://goo.gl/5nXnuu

65. Vicenzino B, Paungmali A, Teys P. Mulligan's mobilization-with-movement, positional faults and pain relief: current concepts from a critical review of literature. Man Ther. 2007; 12: 98-108. Ref.: https://goo.gl/cDdpqr

66. Hearn A, Rivett DA. Cervical SNAGs: a biomechanical analysis. Man Ther. 2002; 7: 71-79. Ref.: https://goo.gl/imQF8R

67. Bialosky J, Bishop M, Robinson M. The Mechanisms of Manual Therapy in the Treatment of Musculoskeletal Pain: A Comprehensive Model. Man Ther. 2009; 14: 531-538. Ref.: https://goo.gl/C2wMYN 
68. Paungmali A, O'Leary S, Souvlis T, Vicenzino B. Hypoalgesic and Sympathoexcitatory Effects of Mobilization with Movement for Lateral Epicondylalgia. Phy Ther. 2003; 83: 374-383. Ref.: https://goo.gl/MRKB5E

69. Tachii R, Sen S, Arfath U. Short-term effect of sustained natural apophyseal glides on cervical joint position sense, pain and neck disability in patients with chronic neck pain. Int $\mathrm{J}$ Therap Rehabilitation Res. 2015; 4: 244-249. Ref.: https://goo.gl/HWQRS1

70. EL-Sayed W, Mohamed A, Abdel-Monem G. Effect of SNAGS Mulligan Technique on Chronic Cervical Radiculopathy: A Randomized Clinical Trial. Med J Cairo. 2017; 85: 787-793. Ref.: https://goo.gl/1mxY1f

71. Carroll L, Hogg-Johnson S, van der Velde G. Course and prognostic factors for neck pain in the general population: results of the Bone and Joint Decade 2000-2010 Task Force on Neck Pain and Its Associated Disorders. Spine. 2008; 33: 75-82. Ref.: https://goo.gl/4qifYQ

72. Chiu T, Leung A. Neck pain in Hong Kong: a telephone survey on prevalence, consequences, and risk groups. Spine. 2006; 31: 540-544. Ref.: https://goo.gl/kRvRQk

73. Goode AP, Freburger J, Carey T. Prevalence, practice patterns, and evidence for chronic neck pain. Arthritis Care Res (Hoboken). 2010; 62: 1594-1601. Ref.: https://goo.gl/JuEu5H

74. Hoving J, de Vet $H$, Twisk J, Deville W, van der Windt $D$, et al. Prognostic factors for neck pain in general practice. Pain. 2004; 110: 639-645. Ref.: https://goo.gl/xqqvjP

75. Kääriä S, Laaksonen $M$, Rahkonen $O$, Lahelma $E$, Leino-Arjas $P$. Risk factors of chronic neck pain: a prospective study among middle-aged employees. Eur J Pain. 2012; 16: 911-920. Ref.: https://goo.gl/H9n5Kh

76. MacDermid JC, Walton DM, Côté $P$, Santaguida PL, Gross A, et al. Use of outcome measures in managing neck pain: an international multidisciplinary survey. Open Orthop J. 2013; 7: 506-520. Ref.: https://goo.gl/yp8JzP

77. Pérez $H$, Perez $J, f$, Martinez A. Is one better than another? A randomized clinical trial of manual therapy for patients with chronic neck pain. Manual Therapy. 2014; 19: 215-221. Ref.: https://goo.gl/NQ3sDg

78. Reid SA, Rivett DA, Katekar MG, Callister R. Sustained natural apophyseal glides (SNAGs) are an effective treatment for cervicogenic dizziness. Manual Therapy. 2008; 13: 357-366. Ref.: https://goo.gl/4yQsu2

79. Thomas L, Rivett DA, Attia J, Parsons M, Levi C. Risk factors and clinical features of craniocervical arterial dissection. Man Ther. 2011; 16: 351-356. Ref.: https://goo.gl/jRNf2f 